\title{
Analysis of Drug Release from Different Agglomerates Using a Mathematical Model
}

\author{
Jiri Petru* and Petr Zamostny \\ Department of Organic Technology, Faculty of Chemical Technology, Institute of Chemical Technology Prague, Technicka 5, 16628 \\ Prague 6, Czech Republic
}

\begin{abstract}
The release rate of an active ingredient from a dosage form may be affected by the agglomeration of an active ingredient before tablet compression. This study is aimed at establishing the effects of the agglomerates on active ingredient release profiles. A model substance, caffeine, was tested in the powdered form and in the two agglomerated forms prepared by wet granulation and compaction. The profiles of caffeine release from these forms were measured using a flow-through cell dissolution apparatus (USP 4) in an open-loop arrangement. To identify the particular processes responsible for the differences in the dissolution profiles of the model forms, we developed a mathematical description of the dissolution profiles. Using this mathematical approach, we proposed that caffeine was adsorbed to the microcrystalline cellulose present in the granulated and compacted form and that diffusion of the active ingredient was inhibited in the presence of a binder in the granulate. Both of these effects resulted in imperfections in the shape of dissolution profiles and in a decrease in the dissolution rate of caffeine. The mathematical model is based on generic parameters, thus it is transferable to other pharmaceutical substances in powdered and agglomerated forms.
\end{abstract}

KEYWORDS: Dissolution; release rate; mathematical modeling; agglomerate; high-shear wet granulation; compaction.

\section{INTRODUCTION}

$\mathrm{M}$ any factors can affect the dissolution of an active pharmaceutical ingredient (API). Since most dosage forms comprise not only the active ingredient but also excipients, the overall rate of API release depends on the properties of both of these components (1-4). Moreover, it also depends on the arrangement and interaction of individual components of the dosage form, which in turn depend on the dosage form processing history, such as a dry or wet agglomeration process.

Several studies dealing with the effect of different granulation methods on the dissolution rate of API show that such a form of processing can heavily influence the dissolution rate (5-9). However, these works differ in their results. These variations are mainly due to the use of different excipients and active substances. The authors of these studies kindly provided us valuable experiences and suggestions, but with regard to current techniques and options, these studies contain some weaknesses, especially with respect to the effect of a particular process.

Due to advances in information technology in recent years, it is possible to use experimental data for mathematical modeling of dissolution and drug delivery. The main benefit of these models is in reducing the number of experiments and associated costs (10). A mathematical approach can help with investigating how dosage form

${ }^{*}$ Corresponding author. variables (e.g., composition, porosity, and hardness) and manufacturing equipment parameters (e.g., granulation period, compaction pressure) affect overall kinetics of the API release. Based on results from mathematical analysis, the best candidates for conducting in vitro experiments can be chosen, which can significantly facilitate the development of a new product (11).

Numerous mathematical theories dealing with dissolution have been described $(10,12,13)$. Most of these studies have focused on mathematical characterization and quantification of drug release from final dosage forms. However, this is basically impossible due to the complexity of the process, and even recent studies still lack accuracy and ease of application (10). A focus on one particulate process affecting the dissolution can bring better understanding and better characterization of the process.

The same approach was used by Frenning and Strømme (14). They proposed a valuable model based on generic parameters, but we decided to focus more on the analysis of the effects that the intermediate agglomerates and their characteristics, instead of the entire tablet, can have on the API release rate. In a recent paper (15), we have shown the API release rate from final dosage forms can be predicted from the dissolution behavior of agglomerates. The characterization of agglomerates is more specific and easier to obtain. Thus, in this study we intended to identify the mechanisms taking place in the dissolution process of agglomerates and quantify them by a regression model. 


\section{MATERIALS AND METHODS \\ Model Substance}

Anhydrous caffeine was used as a model substance. Sieve fractions of powdered, wet-granulated, and compacted caffeine were included in the study.

\section{Powdered Caffeine}

Powdered caffeine was obtained by courtesy of Zentiva Inc. (Czech Republic). Particle size, measured by optical microscopy image analysis (reported as maximum Feret diameter), was $<10 \mu \mathrm{m}$ for $70 \%$ of particles and $<45 \mu \mathrm{m}$ for $95 \%$ of particles. The powdered caffeine was sieved to obtain sieve fractions of 75-125 $\mu \mathrm{m}$ and 425-1000 $\mu \mathrm{m}$ particle diameter for dissolution experiments.

\section{Wet-Granulated Caffeine}

Wet-granulated caffeine was obtained by granulating powdered caffeine, Avicel PH 101 microcrystalline cellulose (FMC BioPolymer, USA), and Povidone 30 (50:48.3:1.7 $\mathrm{w} / \mathrm{w} / \mathrm{w}$ ) in a laboratory high-shear mixer vertical granulator (Glatt VG 25) using purified water. After drying, the granulate was sieved through a $1.25-\mathrm{mm}$ sieve. The resulting granulate was further sieved as necessary to gain the desired sieve fractions for dissolution experiments.

\section{Compacted Caffeine}

Compacted caffeine was obtained by compressing a mixture of powdered caffeine and Avicel PH 101 (50:50 $\mathrm{w} / \mathrm{w}$ ) into 16-mm lenticular tablets (average tablet strength approx. $40 \mathrm{~N}$ ), then processing the tablets on a Frewitt sieving mill using a $1.25 \mathrm{~mm}$-sieve. The resulting granules were further sieved to acquire sieve fractions of 75-125 $\mu \mathrm{m}$ and 425-1000 $\mu \mathrm{m}$ particle diameter for dissolution experiments.

Experiments were carried out using following samples:

$\begin{array}{lcl}\text { Powdered caffeine } & 75-125 \mu \mathrm{m} & \left(\mathrm{P}_{75}\right) \\ \text { Powdered caffeine } & 425-1000 \mu \mathrm{m} & \left(\mathrm{P}_{425}\right) \\ \text { Wet granulated caffeine } & 75-125 \mu \mathrm{m} & \left(\mathrm{G}_{75}\right) \\ \text { Wet granulated caffeine } & 425-1000 \mu \mathrm{m} & \left(\mathrm{G}_{425}\right) \\ \text { Compacted caffeine } & 75-125 \mu \mathrm{m} & \left(\mathrm{C}_{75}\right) \\ \text { Compacted caffeine } & 425-1000 \mu \mathrm{m} & \left(\mathrm{C}_{425}\right)\end{array}$

\section{Dissolution Experiments}

Dissolution tests were carried out using USP Apparatus 4 (Sotax Dissotest CE) with a reciprocating pump (Sotax $\mathrm{CY}, 1$ Sotax, Switzerland), equipped with a 12-mm diameter dissolution cell for powders and granulates. The open-loop configuration was used according to USP (16) and Beyssac and Lavine (17). The dissolution apparatus and dissolution medium $(0.1 \mathrm{M} \mathrm{HCl})$ were both immersed in a $37^{\circ} \mathrm{C}$ water bath.

Samples were taken at the cell outlet tubing. Initially, thirteen samples were withdrawn uniformly in 10-sec intervals. Additional samples were taken at 2.5, 3, 4, 5, 7,
10, 15, and $20 \mathrm{~min}$. All samples were analyzed using high performance liquid chromatography.

Concentration of the model substance in the samples was measured using an LC Prominence system (Shimadzu, Japan) with a PDA detector and a LiChrospher 60, RP-select B, 5- $\mu$ m column (Merck). A solution of dibasic ammonium phosphate in water $(\mathrm{pH}=3.5 \pm 0.1)$ and methanol (1.5:1) was used as the mobile phase (isocratic) at $1 \mathrm{~mL} /$ $\min$. The injection volume of the sample was $2 \mu \mathrm{L}$. ERA 3.0 software (18) was used for all computations and regression analysis of the experimental data (19).

\section{RESULTS AND DISCUSSION}

Results of the dissolution experiments are represented as data points in Figure 1. Each experiment was performed twice, and the data points represent the arithmetic mean of two corresponding points of the replicated experiments for clarity. The differences between replicates were negligible compared with the differences between different samples. It is obvious that the dissolution profiles of different caffeine forms vary.

To analyze these dissolution profiles we propose a mathematical model.

\section{Mathematical Model}

The model describing the change of concentration over time was based on a modified Noyes-Whitney equation (20) under sink conditions:

$$
\frac{d c}{d t}=-k \frac{A}{V} C_{s}
$$

or expressed as the change of weight over time:

$$
\frac{d m}{d t}=-\frac{D}{\delta_{H L}} A C_{s}
$$

where $k$ is the dissolution rate constant, $C_{s}$ is the saturated solution concentration, $A$ is the interfacial area on which dissolution takes place (in this case the surface of all particles is assumed), $V$ is the volume in which the dissolution takes place (volume of the flow-through cell), $D$ is the diffusion coefficient of caffeine, and $\delta_{H L}$ is the hydrodynamic layer thickness. A model based only on this equation, where the dissolution rate constant with area and volume factors was the adjustable parameter, was fitted to the experimental data, and the optimal value of the adjustable parameter was estimated by a nonlinear regression analysis in ERA software. The sum of squared residual errors, weighted by the reciprocal value of respective dependent variable $(f)$, was used as an objective function. Results of this preliminary approach are shown in Figure 1. It is obvious from the figure that this equation cannot be used alone, since it does not reflect the effects of the agglomerated particle and its components on the release rate of caffeine. The minimum value of the objective func- 


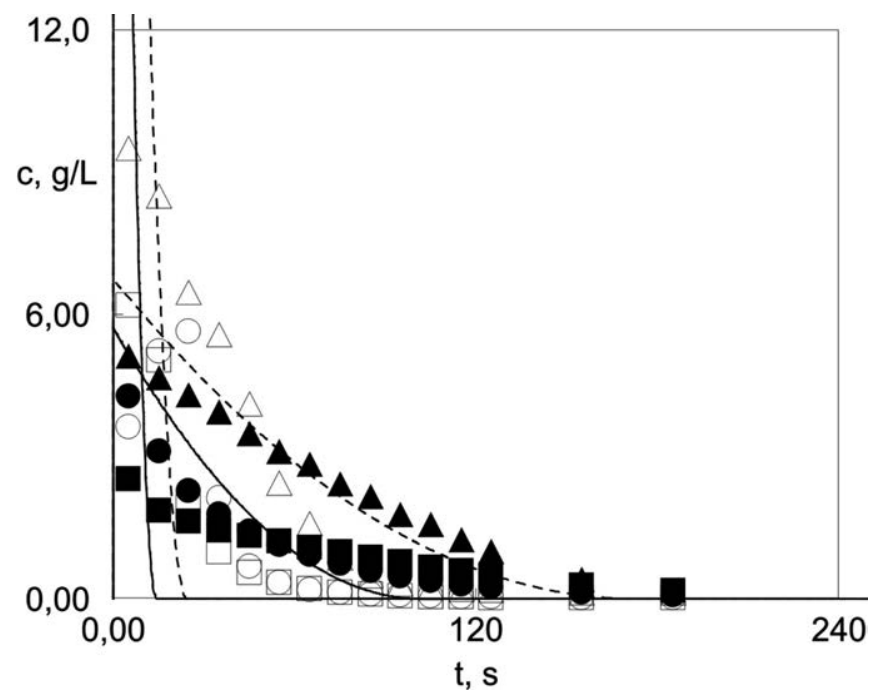

Figure 1. Measured (points) and calculated (lines; using eq 2) dissolution profiles of ( $\Delta$, dashed line) powdered caffeine, $(\square$, dotted line) granulated caffeine, ( 0 , solid line) compacted caffeine of 425-1000 $\mu \mathrm{m}$ (filled) and 75-125 $\mu \mathrm{m}$ (empty).

tion $f$, which was obtained by fitting the dissolution data using eq 2, was as high as 44.69 .

Before we began to deal with properties of excipients and their influence on caffeine release, we had to disprove that the change in the dissolution profiles of the agglomerates was caused by the change of properties of caffeine itself due to the agglomeration. Particularly, we were concerned with a polymorphic transition of caffeine. This was eliminated on the basis of results from X-ray powder diffraction analysis.

Hence, further analysis of experimental data was performed with respect to the influence of excipients on caffeine release from agglomerates. The deviations from the Noyes-Whitney model behavior and the observations of the dissolution allowed us to propose the effects responsible for the modified release profile. Those included the resistance to diffusion caused by the insoluble excipient matrix, the adsorption of caffeine on the excipient surface, and the rate of wetting of the sample surface. These effects were progressively incorporated into eq 2 . This mathematical analysis presents an indirect proof of the proposed processes, and an independent analytical measurement would be necessary to confirm our assumptions.

At first, the resistance to diffusion term was expressed (eq 3). It involves the mass transfer through the hydrodynamic layer, which is constant at steady hydrodynamic conditions, and the transfer trough the excipient matrix, which decreases over time (discussed later). Both the hydrodynamic layer and the matrix have different diffusion coefficients as the resistance to diffusion for both barriers is different.

$$
\frac{d m}{d t}=-\frac{A C_{s}}{\frac{\delta_{H L}}{D}+\frac{d_{p 0}-d_{p}}{2 D_{e f}}}
$$

$D_{e f}$ is the effective diffusion coefficient of caffeine in the leached particle residue, which characterizes the increase of the resistance to diffusion, and $d_{p}$ and $d_{p 0}$ are actual and initial particle diameters, respectively.

Next, the adsorption term was added (eq 4). The adsorption term was based on the Langmuir equation (21)

$$
\frac{d m}{d t}=-\frac{A C_{s}}{\frac{\delta_{H L}}{D}+\frac{d_{p 0}-d_{p}}{2 D_{e f}}}+k_{A d s} \frac{K_{A d s} m}{1+K_{A d s} m}
$$

where $k_{A d s}$ is the rate of adsorption, $K_{\text {Ads }}$ is the adsorption coefficient, and $m$ is the weight of caffeine remaining in the sample.

Finally, the wetting term was added to describe the increasing dissolution rate caused by relatively slow (rapid but not instantaneous) wetting of agglomerates in the dissolution cell, and eq 5 was obtained.

$\frac{d m}{d t}=-\left(\frac{A C_{s}}{\frac{\delta_{H L}}{D}+\frac{d_{p 0}-d_{p}}{2 D_{e f}}}+k_{A d s} \frac{K_{A d s} m}{1+K_{A d s} m}\right) \alpha(1-\alpha) \frac{m}{\beta+m}$

Wetting was observed during the experiment, only in the first seconds of the experiment, and it occurred mainly with 75-125 $\mu \mathrm{m}$ particles.

We had to include the description of the wetting in the model to be able to evaluate the dissolution for the entirety of the experiment without the exclusion of the very first points of the dissolution profiles. It was not the purpose of this paper to study or characterize this effect in detail, and thus we chose an empirical description, which enabled us to evaluate the experiments with the elimination of the influence of wetting. Hence, the kinetics of wetting was characterized by constants $\alpha$ and $\beta$ for each sample based upon the fitting of the dissolution data. After the constants $\alpha$ and $\beta$ were determined, they were no longer used as adjustable parameters.

Subsequently, the area and the diameter from the weight of the sample (eqs 6 and 7) were expressed, and the general model, eq 8 , was obtained under the assumption of roughly uniform shape of all particles.

$$
\begin{gathered}
A=N K_{\mathrm{A}} d_{p}^{2} \\
d_{p}=\sqrt[3]{\frac{m}{N K_{\mathrm{V}} \rho}}
\end{gathered}
$$




$$
\begin{gathered}
\frac{d m}{d t}=\left(-\frac{C_{s} N^{1 / 3} K_{\mathrm{A}}\left(\frac{m}{\rho K_{\mathrm{V}}}\right)^{2 / 3}}{\frac{\delta_{H L}}{D}} \frac{1}{1+\frac{\left(\rho K_{\mathrm{V}} N\right)^{-1 / 3}\left(m^{1 / 3}+m_{0}^{1 / 3}\right)}{2 D_{e f} \frac{\delta_{H L}}{D}}}\right. \\
\left.+k_{\text {Ads }} \frac{K_{\text {Ads }} m}{1+K_{\text {Ads }} m}\right) \alpha(1-\alpha) \frac{m}{\beta+m}
\end{gathered}
$$

where $N$ is the number of particles, and $K_{A}$ and $K_{V}$ are surface and volume factors, respectively.

Adjustable parameters (i.e., parameters that characterize the process and vary from one form to another) were

$$
\begin{gathered}
p_{1}=\frac{K_{\mathrm{V}}{ }^{2 / 3}}{K_{\mathrm{A}}} \frac{\delta_{H L}}{D}, \\
p_{2}=\frac{1}{D_{e f} \frac{\delta_{H L}}{D} K_{\mathrm{V}}{ }^{1 / 3}}
\end{gathered}
$$

$k_{\text {Ads }}$ and $K_{\text {Ads }}$. These parameters were estimated by fitting the data in ERA software using the simultaneous regression of all data sets, all sharing the same value of the $p_{1}$ parameter, which is independent of the agglomerate type under the assumption that the shape factors of all agglomerate types are roughly identical.

The adsorption rate constant for the powdered caffeine was insignificantly deviated from zero, which corresponds to the absence of any excipient onto which the caffeine would adsorb. In addition, the parameter characterizing the resistance to diffusion was insignificant for powdered caffeine. Such parameters, the values of which were statistically insignificant at the $95 \%$ confidence level, were excluded from the model.

Consequently, parameters were tested whether their values depend on the type of agglomerate or on the size of particles in the sample. Only when an insignificant increase of the objective function value was observed could parameters have been lumped (combined). The maximum objective function increase that is not statistically significant was calculated according to Himmelblau (22) and used in a different problem (23).

Technically, this was done by calculating the $95 \%$ confidence limits for all parameters initially. Then, if two parameters representing the same property for the different type of agglomerate had their values mutually positioned within the confidence limits of the other parameter, they may be lumped together because their difference was statistically insignificant at the $95 \%$ confidence level.
Hence, the regression analysis showed that the lower $95 \%$ confidence limits for $p_{2, P 75}, p_{2, P 425}, k_{A d s, P 75}, k_{A d s, P 425}$, $K_{A d s, P 75}$, and $K_{A d s, P 425}$ parameters are limiting to zero. It may be due to either the parameter being insignificant or being correlated to another insignificant parameter. The necessary parameter reduction was done in a one-by-one approach, excluding the parameter with the widest confidence interval and recalculating the regression analysis for the model comprising $N-1$ parameters.

Consequently, results of the regression analysis show that the adsorption coefficient $K_{\text {Ads }}$ is independent of the particle size, and the rate of adsorption $k_{A d s}$ is common for all agglomerated forms, but the adsorption is proportional to the initial average surface area of the sample. Hence, the value of the initial surface area $\left(A_{0}\right)$ had to be introduced in the model for each form, and a new adjustable parameter $\mu_{\text {Ads }}$ was implemented according to the equation

$$
k_{A d s}=A_{0} \mu_{A d s}
$$

Then the final form of the equation used for the subsequent analysis was

$$
\begin{aligned}
\frac{d m}{d t}= & \left(-\frac{C_{s} N^{1 / 3} K_{\mathrm{A}}\left(\frac{m}{\rho K_{\mathrm{V}}}\right)^{2 / 3}}{\frac{\delta_{H L}}{D}} \frac{1}{1+\frac{\left(\rho K_{\mathrm{V}} N\right)^{-1 / 3}\left(m^{1 / 3}+m_{0}^{1 / 3}\right)}{2 D_{e f} \frac{\delta_{H L}}{D}}}\right. \\
& \left.+A_{0} \mu_{A d s} \frac{K_{A d s} m}{1+K_{A d s} m}\right) \alpha(1-\alpha) \frac{m}{\beta+m}
\end{aligned}
$$

$K_{A d s, C 75}$ and $K_{A d s, C 425}$ as well as $K_{A d s, G 75}$ and $K_{A d s, G 425}$ were lumped into $K_{A d s, C}$ and $K_{A d s, G}$, respectively, and $k_{A d s, C 75,}$ $k_{A d s, C 425}, k_{A d s, G 75}$, and $k_{A d s, G 425}$ were lumped together into the single parameter $\mu_{A d s}$.

In summary, the number of parameters was substantially reduced from nineteen to eight by parameter exclusion and lumping, and the objective function increased very slightly from 2.39 to 3.91 as a result. The method of the following parameter reduction is shown below.

Parameter estimates in this phase of model identification and their reliability indicated by the confidence intervals are given in Table 1.

It is important to note that the values of $p_{2}$ parameter for the compacted caffeine are insignificant unlike its values for granulated caffeine, thus showing that the effect of dissolution inhibition by an excipient depends strictly on the excipient nature. Since the compacted sample did not contain any binder, thus neither binder bridges, 
it can be concluded that the resistance to diffusion is given mainly by the presence of a binder. The negligible $p_{2}$ values for compacts show the excipient matrix disintegrated at the same rate as the caffeine dissolved and did not present any barrier for further caffeine dissolution. The large $p_{2}$ values for granules show that the Avicel/Povidone matrix remained substantially intact, and the caffeine had to be "leached" from the matrix through an increasingly thicker barrier.

Another modification of the model could be made after noticing that confidence intervals of adsorption coefficients $K_{A d s, C}$ and $K_{A d s, G}$ intersect. Thus, those parameters can be lumped into single $K_{\text {Ads }}$ parameter. That is, the adsorption of active compound to the surface of the excipient is, in this case, mainly a function of the microcrystalline cellulose content, which is in agreement with $(3,14)$.

A final estimation of parameters is given in the Table 2 .

From a comparison of the values of parameter $p_{2}$, it is obvious that the resistance to diffusion is much higher for the sample of smaller particles. This is in agreement with Griffith theory (24), according to which all materials contain flaws that cause them to break. From the point of dissolution, these flaws are places where diffusion can take place more rapidly. The concentration factor of the flaws increases as the size of particles in the sample decreases. Thus, it can be observed in Table 2 that parameter $p_{2}$ characterizing the resistance to diffusion is more than five times greater for smaller particles than for larger particles.

Table 1. Estimated Parameters and Confidence Intervals for Model (eq 10)

\begin{tabular}{|c|c|c|}
\hline Parameter & Value & Confidence interval (95\%) \\
\hline$p_{1}, \mathrm{dm} \mathrm{s}^{-1}$ & 488 & $<446 ; 525>$ \\
\hline$p_{2, \mathrm{c} 75, \mathrm{dm}}$ & 0.0038 & $<0 ; 321>$ \\
\hline$p_{2, C 425,} \mathrm{dm}$ & 0.0043 & $<0 ; 86>$ \\
\hline$p_{2, G 75,} \mathrm{dm}$ & 958 & $<311 ; 1542>$ \\
\hline$p_{2, \mathrm{G} 425, \mathrm{dm}}$ & 173 & $<54 ; 288>$ \\
\hline$\mu_{A d s}, \mathrm{gdm}^{-2} \mathrm{~s}^{-1}$ & 0.0045 & $<0.0035 ; 0.0068>$ \\
\hline$K_{A d s, c}, g^{-1}$ & 228 & $<144 ; 468>$ \\
\hline$K_{A d s, G,} g^{-1}$ & 174 & $\langle 86 ; \infty\rangle$ \\
\hline
\end{tabular}

Table 2. Final Estimation of Parameters and Confidence Intervals after Parameter-Lumping Procedure

\begin{tabular}{ccc}
\hline Parameter & Value & Confidence interval (95\%) \\
\hline$p_{1}, \mathrm{dm} \mathrm{s}^{-1}$ & 488 & $<468 ; 511>$ \\
\hline$p_{2, G 75,} \mathrm{dm}$ & 991 & $<641 ; 1428>$ \\
\hline$p_{2, G 425,} \mathrm{dm}$ & 174 & $<87 ; 267>$ \\
\hline$\mu_{A d s,} \mathrm{~g} \mathrm{dm}^{-2} \mathrm{~s}^{-1}$ & 0.0045 & $<0.0036 ; 0.0058>$ \\
\hline$K_{A d s}, g^{-1}$ & 230 & $<180 ; 320>$ \\
\hline
\end{tabular}

However, higher resistance to diffusion of the small granulate particles does not mean that the overall rate of diffusion is lower. This effect has much less influence than the fact that dissolution rate is proportional to the surface on which it takes place (in the model given by the number, density, and weight of particles). Therefore, smaller particles always dissolve faster than larger particles. This corresponds with profiles in Figure 2.

The dissolution profiles presented in Figure 2 were calculated using the parameters in Table 2 . This figure shows that the estimation of parameters and the proposal of occurring physical processes was successful, as the calculated profiles correlate very well with the experimental data.

\section{Specific Dissolution Rate}

For better assessing the differences in the dissolution of caffeine in the model forms, the specific rate of dissolution was calculated using the model and the values of the parameters above. The specific rate of dissolution is an indicator of the dissolution behavior of a unit amount of a dissolved sample. It is obtained by dividing the rate of dissolution by the actual weight of caffeine the sample.

After focusing on the dependence of the specific dissolution rate on time (Figure 3 ), it is visible at first glance that smaller particles of the same form dissolve faster than larger particles, which was already discussed.

More interesting in Figure 3 is the comparison of the agglomerates and powdered caffeine. While the specific dissolution rate of the powdered caffeine grows to infinity in time, for granulated forms it drops to zero. The profiles of the powdered forms show the behavior of a theoretically

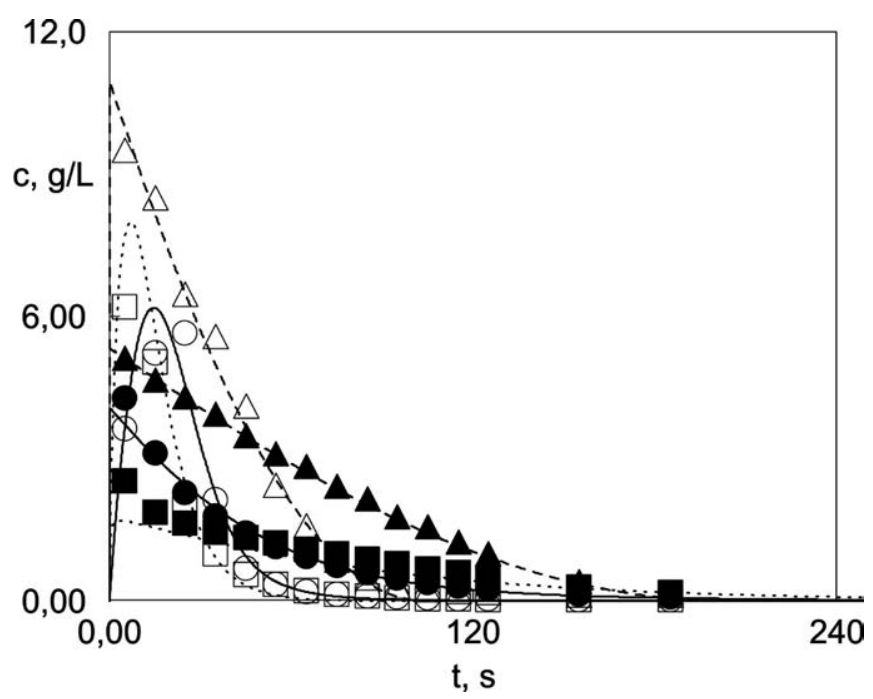

Figure 2. Measured (points) and calculated (lines) dissolution profiles of $(\Delta$, dashed line) powdered caffeine, ( $\square$, dotted) wet granulate, $(0$, solid) dry granulate of 425-1000 $\mu \mathrm{m}$ (filled markers) and 75-125 $\mu \mathrm{m}$ (empty) after lumping procedure. 


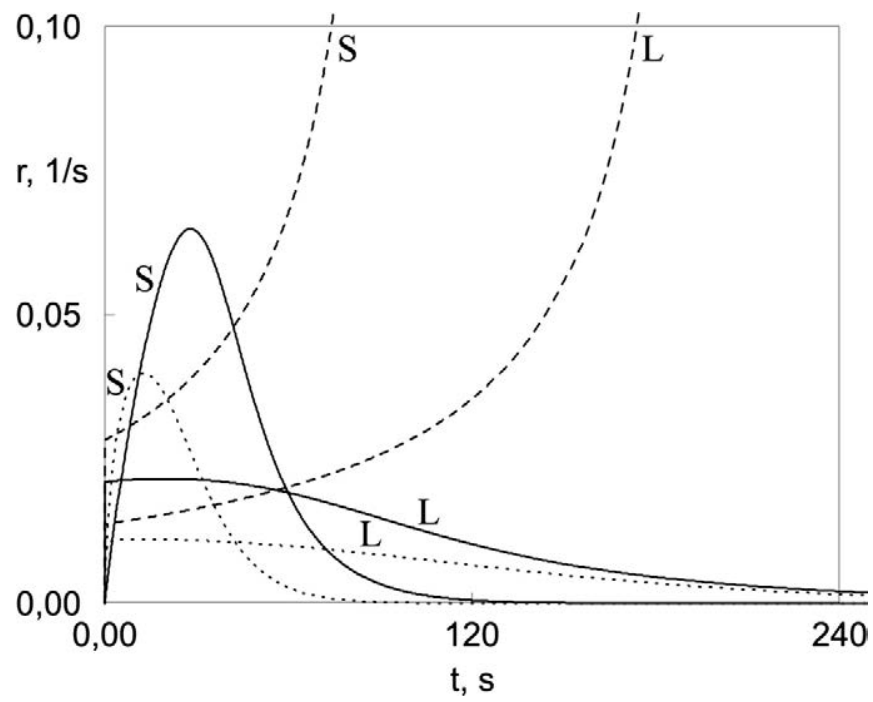

Figure 3. Specific rate of caffeine dissolution in (...) granulated, (-) compacted, and (- - -) powdered caffeine containing particle sizes of 75-125 $\mu \mathrm{m}$ (S) and 425-1000 $\mu \mathrm{m}(\mathrm{L})$.

ideal mixture, which follows the Noyes-Whitney equation. Because the dissolution of these mixtures is not decelerated by any effect, the specific rate grows to infinity as the fraction between the surface and volume increases during the dissolution. On the contrary, the specific rate of granulated forms is not growing during the dissolution because the adsorption of caffeine to microcrystalline cellulose occurs.

The comparison of the granulated forms is shown in Figure 3. The shape of the curves for which the adsorption is responsible is relatively similar. The difference is in the absolute values of the specific rate. Smaller values of the dissolution rate of the granulates are caused by the presence of the binder, thus resistance to diffusion, which is not occurring in compacted caffeine. Therefore, the compacted caffeine dissolved faster than granulated caffeine.

\section{Mathematical Model as a Prediction Tool}

Originally, we did not intend to develop a mathematical model for predicting the dissolution behavior, but we meant to use the mathematical description to quantify changes caused by the agglomeration of the active ingredient and discuss the differences between the tested forms. However, the final form of the model allows using the model also as a prediction tool for agglomerated caffeine. Therefore, we measured the independent experiments (i.e., the dissolution profiles of the agglomerated forms) but with different particle sizes $(250-425 \mu \mathrm{m})$. The independent experimental data were described by the model using the adjustable parameter values above. Except for granulated caffeine, parameters characterizing the resistance to diffusion had to be adjusted, as the resistance to diffusion is related to the particle size. The value

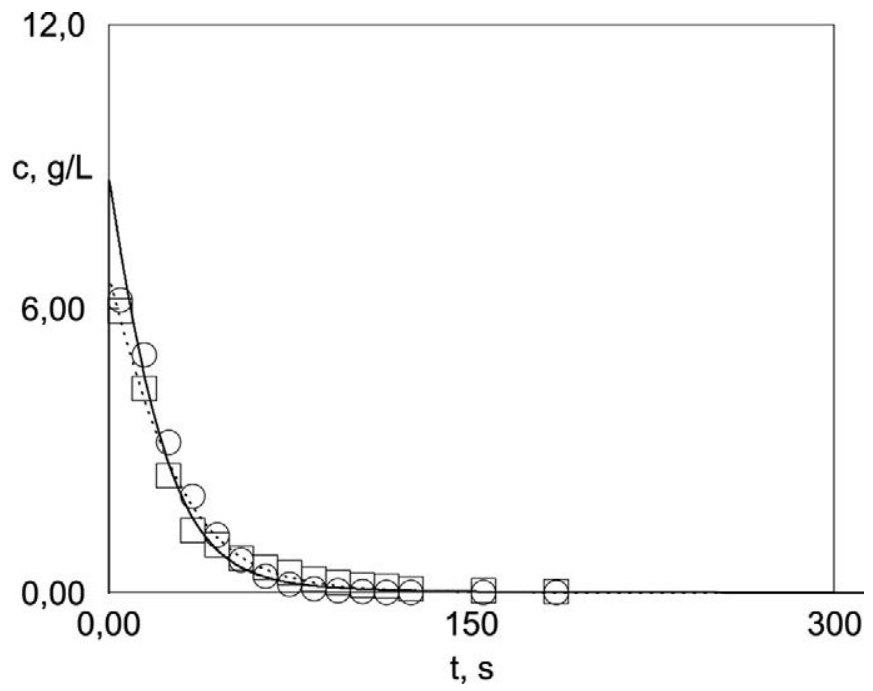

Figure 4. Measured (points) and calculated (lines) dissolution profiles of ( 0 , solid line) compacted and ( $\square$, dotted line) granulated caffeine of 250-425 $\mu m$.

of the parameter $p_{2}$ was $163 \mathrm{dm}$. Thus, with $95 \%$ probability it is between $p_{2, G 75}$ and $p_{2, G 425}$, very close to parameter $p_{2, \mathrm{G} 425}$ (Table 2), which is in agreement with the theory of flaws discussed above.

Our model fits the independent experimental data very well (Figure 4). The model is fully predictive for the compacted caffeine with different particle sizes. For the granulated caffeine, one parameter had to be calculated to adjust the model to the experimental data. Thus, the model is not fully predictive in this case, but the magnitude of caffeine release inhibition caused by the binder can be expressed.

\section{CONCLUSIONS}

We have shown that the flow-through cell dissolution apparatus provides data well suited to mathematical modeling and to precise investigation of API release inhibition caused by an excipient or by the application of an agglomerating technique.

Our proposed model, extending the Noyes-Whitney equation with both the assumption of diffusion layer and a monolayer adsorption equation, was able to fit the experimental data extremely well. The model and its specific development procedure can help in understanding processes that, despite being within the sink conditions, cause one or more of the following: nonideal shape of the dissolution profile, deceleration of the dissolution rate, and incomplete dissolution of an active ingredient. The model is based on generic parameters; hence, it is transferable to agglomerates with different compositions and allows for computer aided design of the dosage form.

Our model appears highly promising, particularly due to its simplicity. Thus in our next study, it will be further 
investigated especially in terms of the dependence of excipient amount on adjustable parameter values.

\section{ACKNOWLEDGMENT}

Financial support was received from specific university research (MSMT No 20/2013).

\section{SYMBOLS}

A interfacial area, $\mathrm{m}^{2}$

c concentration, $\mathrm{kg} \mathrm{m}^{-3}$

d diameter, $\mathrm{m}$

D diffusion coefficient, $\mathrm{m}^{2} \mathrm{~s}^{-1}$

$\mathrm{k}$ rate constant, $\mathrm{m} \mathrm{s}^{-1}$ (dissolution)/ $\mathrm{kg} \mathrm{s}^{-1}$ (adsorption)

$\mathrm{K}$ coefficient

$\mathrm{m}$ weight, $\mathrm{kg}$

$\mathrm{N}$ number

$\mathrm{p}$ parameter

$\mathrm{t}$ time, $\mathrm{s}$

$\checkmark$ volume of dissolution apparatus, $\mathrm{m}^{3}$

\section{Greek letters}

$\alpha$ wetting constant

$\beta$ wetting constant

$\delta_{H L}$ hydrodynamic layer thickness, $\mathrm{m}$

$\rho$ density, $\mathrm{kg} \mathrm{m}^{-3}$

$\mu \quad$ rate constant, $\mathrm{kg} \mathrm{m} \mathrm{s}^{-1}$

Subscripts
$0 \quad$ initial
A surface
Ads adsorption
ef effective
p particle
S saturated solution
V volume
C compacted
G granulated

\section{REFERENCES}

1. Levy, G.; Antkowiak, J. M.; Procknal, J. A.; White, D. C. Effect of certain tablet formulation factors on dissolution rate of the active ingredient. II. Granule size, starch concentration, and compression pressure. J. Pharm. Sci. 1963, 52 (11), 1047-1051. DOI: 10.1002/ jps.2600521106.

2. Williams, R. O.; Reynolds, T. D.; Cabelka, T. D.; Sykora, M. A.; Mahaguna, V. Investigation of Excipient Type and Level on Drug Release from Controlled Release Tablets Containing HPMC. Pharm. Dev. Technol. 2002, 7 (2), 181-193. DOI: 10.1081/PDT-120003486.

3. Senderoff, R. I.; Mahjour, M.; Radebaugh, G. W. Characterization of adsorption behavior by solid dosage form excipients in formulation development. Int. J. Pharm. 1992, 83 (1-3), 65-72. DOI: 10.1016/03785173(82)90009-6.
4. Wagner, J. G. Factors affecting rate of dissolution of drugs from tablets and capsules and interpretation of dissolution rate data from in vitro testing of tablets and capsules. Drug Intel. Clin. Pharm. 1970, 4, 132-137.

5. Unvala, H. M.; Schwartz, J. B.; Schnaare, R. L. The Effect of the Wet Granulation Process on Drug Dissolution. Drug Dev. Ind. Pharm. 1988, 14 (10), 1327-1349. DOI: $10.3109 / 03639048809151936$.

6. Ghorab, M. K.; Adeyeye, M. C., Enhancement of Ibuprofen Dissolution via Wet Granulation with $\beta$-Cyclodextrin. Pharm. Dev. Technol. 2001, 6 (3), 305314. DOI: 10.1081/PDT-100002611.

7. Solvang, S.; Finholt, P. Effect of tablet processing and formulation factors on dissolution rate of the active ingredient in human gastric juice. J. Pharm. Sci. 1970, 59 (1), 49-52. DOI: 10.1002/jps.2600590106.

8. Bremer, P. O.; Kolstad, B.; Finholt, P. Direct compression of tablets. Medd. Nor. Farm. Selsk. 1969, 31 (7), 67-81.

9. Inghelbrecht, S.; Remon, J. P. Roller compaction and tableting of microcrystalline cellulose/drug mixtures. Int. J. Pharm. 1998, 161 (2), 215-224. DOI: 10.1016/ S0378-5173(97)00356-6.

10. Siepmann, J.; Siepmann, F. Mathematical modeling of drug delivery. Int. J. Pharm. 2008, 364 (2), 328-343. DOI: 10.1016/j.ijpharm.2008.09.004.

11. Khan, M. A.; Shefeeq, T. Role of Mathematical Modeling in Controlled Drug Delivery. J. Sci. Res. 2009, 1 (3), 539-550.

12. Morrison, A. B.; Campbell, J. A. Tablet disintegration and physiological availability of drugs. J. Pharm. Sci. 1965, 54 (1), 1-8. DOI: 10.1002/jps.2600540102.

13. Dokoumetzidis, A.; Macheras, P. A century of dissolution research: From Noyes and Whitney to the Biopharmaceutics Classification System. Int. J. Pharm. 2006, 321 (1-2), 1-11. DOI: 10.1016/j. ijpharm.2006.07.011.

14. Frenning, G.; Strømme, M. Drug release modeled by dissolution, diffusion, and immobilization. Int. J. Pharm. 2003, 250 (1), 137-145. DOI: 10.1016/S03785173(02)00539-2.

15. Petru, J.; Zamostny, P. Prediction of Dissolution Behavior of Final Dosage forms Prepared by Different Granulation Methods. Procedia Eng. 2012, 42, $1463-$ 1473. DOI: 10.1016/j.proeng.2012.07.539.

16. $<711>$ Dissolution. In The United States Pharmacopeia and National Formulary USP 34-NF 29; The United States Pharmacopeial Convention, Inc.: Rockville, MD, 2011.

17. Beyssac, E.; Lavigne, J. Dissolution Study of Active Pharmaceutical Ingredients Using the Flow Through Apparatus USP 4. Dissolution Technol. 2005, 12 (2), 23-25.

18. Easy Regression Analysis Web site. http://www.vscht. cz/kot/era/ (accessed Oct 5, 2014). 
19. Zamostny, P.; Belohlav, Z. A software for regression analysis of kinetic data. Comput. Chem. (Oxford, U.K.) 1999, 23 (5), 479-485. DOI: 10.1016/S00978485(99)00024-8.

20. Noyes, A. A.; Whitney, W. R. The rate of solution of solid substances in their own solutions. J. Am. Chem. Soc. 1897, 19 (12), 930-934. DOI: 10.1021/ja02086a003.

21. Langmuir, I. The constitution and fundamental properties of solids and liquids. Part I. Solids. J. Am. Chem. Soc. 1916, 38 (11), 2221-2295. DOI: 10.1021/ja02268a002.
22. Himmelblau, D. M. Process Analysis by Statistical Methods; Wiley: New York, 1970.

23. Belohlav, Z.; Zamostny, P.; Kluson, P.; Volf, J. Application of random-search algorithm for regression analysis of catalytic hydrogenations. Can. J. Chem. Eng. 1997, 75 (4), 735-742. DOI: 10.1002/cjce.5450750411.

24. Griffith, A. A. The phenomena of rupture and flow in solids. Philos. Trans. R. Soc., A 1921, 221, 163-198. DOI: 10.1098/rsta.1921.0006. 